\title{
La políitica de discapacidad del sector seguridad y defensa en Colombia: una mirada desde los derechos fundamentales*
}

The Policy on Disability in the Colombian Defense and Security Sector: A View Based on Fundamental

Rights

A Política de Deficiência do Setor Segurança e Defesa na Colômbia: Um Olhar desde os Direitos

Fundamentais

Martha Adriana Crespo Carrillo Ft.'

Recibido: 9 de mayo de $2017 \bullet$ Aprobado: 22 de junio de 2017

Doi: http://dx.doi.org/10.12804/revistas.urosario.edu.co/revsalud/a.6126

Para citar este artículo: Crespo MA. La política de discapacidad del sector seguridad y defensa en Colombia: una mirada desde los derechos fundamentales. Rev Cienc Salud. 2017;15(3):427-440. Doi: http://dx.doi.org/10.12804/revistas.urosario.edu.co/revsalud/a.6126

\section{Resumen}

Introducción: la política de discapacidad del sector seguridad y defensa en Colombia tiene como objetivo la implementación del Sistema de Gestión del Riesgo y Rehabilitación Integral (SGRRI) para articular las acciones de los diferentes actores implicados en el sistema. Este artículo reflexiona sobre la construcción e implementación de esta política como un instrumento para avanzar hacia la inclusión social y garantizar el goce de los derechos fundamentales de las personas de la fuerza pública con discapacidad ( $\mathrm{PcD}$ ). Desarrollo: en Colombia la visión y manejo de la discapacidad se ha transformado a partir de la Constitución Política de 1991. El Ministerio de Defensa Nacional (MDN) no ha sido ajeno a estos cambios y actualmente implementa la política de discapacidad para la fuerza pública, que es una iniciativa destacable en el contexto de América Latina. Dicha política, si bien tiene su fundamento en el modelo social, presenta limitaciones en su implementación para la garantía de los derechos humanos, lo cual implica avanzar hacia un esfuerzo articulador que permita la plena inclusión de este colectivo. Conclusiones: la política de discapacidad del sector seguridad y defensa ha sido un instrumento para avanzar hacia la inclusión social y el goce de los derechos fundamentales de las PcD de la fuerza pública. A pesar de las limitaciones, se evidencia

\footnotetext{
* El presente artículo es presentado como opción de grado para obtener el título de Magíster en Derechos Humanos y Derecho Internacional de los Conflictos Armados de la Escuela Superior de Guerra, dentro del proyecto de investigación "Estrategias para enfrentar los retos de la sociedad y de las Fuerzas Militares de Colombia para la construcción y consolidación de la paz: Derechos Humanos y DICA como instrumento" vinculado al grupo de investigación en DDHH, DICA y justicia inscrito en Colciencias.

1 Ministerio de Defensa Nacional. Centro de Rehabilitación Inclusiva. Correo electrónico: adrianitacrespo@gmail.com
} 
un esfuerzo por garantizar el pleno goce de derechos como la salud, la educación, el empleo y la participación en política, entre otros, cuyo primer resultado es la implementación del SGRRI.

Palabras clave: discapacidad, políticas públicas, derechos fundamentales, fuerza pública.

\section{Abstract}

Introduction: The aim of disability policy in the Colombian defense and security sector is to implement the Risk Management and Integral Rehabilitation System (SGRRI for its initials in Spanish) and to coordinate the actions of the different actors involved. This article reflects on the construction and implementation of the policy as an instrument to advance social inclusion and to guarantee disabled veterans' enjoyment of human rights. Development: The vision on disability in Colombia has changed profoundly since the adoption of the 1991 Constitution. The Ministry of Defense has not been immune from these changes, and the Armed Forces' disability policy being implemented today is a remarkable initiative in the Latin American context. The policy is grounded in a social model, and while incomplete in its implementation, its goal is to guarantee human rights and promote the social inclusion of its target cohort. Conclusion: The disability policy of the Colombian defense and security sector has been an instrument for promoting social inclusion and the enjoyment of human rights by disabled veterans. Progress has been made despite current limitations to the full enjoyment of elemental rights including health, education, work, and political participation. It is clear that the effort of Ministry of Defense has produced its first results with the implementation of the policy made visible through the work of the SGRRI.

Keywords: Disability, public policy, human rights, Armed Forces.

\section{Resumo}

Introdução: a política de deficiência do setor segurança e defesa na Colômbia tem como objetivo a implementação do Sistema de Gestão do Risco e Reabilitação Integral (SGRRI) para articular as ações dos diferentes atores implicados nele. Este artigo reflete sobre a construção e implantação desta política, como instrumento para avançar à inclusão social e garantir o gozo dos direitos fundamentais das pessoas com deficiência $(\mathrm{PcD})$ da Força Pública. Desenvolvimento: na colômbia a visão e gestão da deficiência se tem transformado a partir da Constituição Política de 1991. O Ministério de Defesa Nacional (MDN) não tem sido alheio a estas mudanças e atualmente implementa a política de deficiência para a Força Pública, que é uma iniciativa destacável no contexto da América Latina. Dita política, se bem tem o seu fundamento no modelo social, apresenta limitações na sua implementação para a garantia dos Direitos Humanos, o qual implica avançar a um esforço articulador que permita a plena inclusão deste coletivo. Conclusões: a política de deficiência do setor segurança e defesa tem sido um instrumento para avançar à inclusão social e o gozo dos direitos fundamentais das pessoas com deficiência da Força Pública. Apesar das limitações, evidencia-se um esforço para garantir o gozo pleno de direitos como a saúde, a educação, o emprego e a participação em política, entre outros, e exemplo disso é a implementação da política mediante a articulação do SGRRI.

Palavras-chave: deficiência, políticas públicas, direitos fundamentais, força pública. 


\section{Introducción}

La situación de las personas con discapacidad (PcD) de la fuerza pública en Colombia ha presentado un cambio visible desde el año 2009, con la aprobación del documento técnico Conpes 3591 (1) que contiene los lineamientos, recomendaciones y proyectos para que el Sistema de Rehabilitación Integral mejore las condiciones de vida de los miembros de la fuerza pública desde la prevención de la discapacidad hasta la inclusión social.

Según las estadísticas del Ministerio de Defensa Nacional (MDN) ${ }^{2}$ la población con discapacidad en la fuerza pública está representada en su gran mayoría por el Ejército Nacional y la Policía Nacional (79\%), lo que refleja que este personal está expuesto a un mayor riesgo por el cumplimiento de la misión institucional. En el Ejército el origen de la discapacidad se generó en un $44,1 \%$ por conflicto armado, en un $19,1 \%$ por enfermedad en general, un $17,8 \%$ por accidente y un $19 \%$ por otras causas (2).

En relación con la discapacidad adquirida en servicio activo, el MDN reporta 9576 uniformados, equivalentes al 2,18\% de los miembros de la fuerza pública vinculados al conflicto armado y expuestos a los riesgos propios de la ocupación del militar o policía (2). El 62\% de estos casos (5938) corresponde a discapacidad motora, seguida de cognitiva con un $17 \%$ (1627 casos), mental 9\% (862 casos), múltiple $7 \%$ (670 casos) y sensorial 5\% (479 casos). Las estructuras que con mayor frecuencia se ven comprometidas en miembros de la fuerza pública son las del sistema musculoesquelético, seguidas por las del sistema nervioso, cardiovascular, inmunológico, respiratorio y visual (2).

2 Por ser un documento interno de trabajo del MDN, este no se encuentra publicado. Puede ser consultado en los archivos del Centro de Rehabilitación Inclusiva (CRI).
Así que el tema de la discapacidad para la fuerza pública no es menor y amerita una reflexión de la cual se ocupa el presente artículo. El eje de análisis gira sobre la construcción e implantación de la política de discapacidad del sector seguridad y defensa como instrumento para avanzar hacia la inclusión social y garantizar el goce de los derechos fundamentales de las PcD de la fuerza pública. Las categorías analizadas fueron discapacidad, políticas públicas $\mathrm{y}$ derechos fundamentales.

Vale la pena precisar que esta política, firmada en el año 2014, es el resultado del arduo trabajo de un equipo que durante más de cinco años desarrolló el Sistema de Gestión de Riesgo y Rehabilitación Integral (SGRRI) en el cual se definieron objetivos y actores para cada fase, como respuesta a los cambios mundiales y a los compromisos adquiridos por Colombia en convenciones internacionales relacionados con el goce efectivo de los derechos de las PcD.

\section{Desarrollo}

El análisis se encuentra dividido en dos partes, la primera señala los antecedentes que soportan la visión actual de la discapacidad en la fuerza pública; la segunda analiza la planeación, implementación e impacto de la política en los derechos humanos de las PcD de la fuerza pública.

Antecedentes de la actual visión de discapacidad en la fuerza pública El concepto de discapacidad es histórico y social. Desde el surgimiento del modelo de prescindencia en la Edad Media atravesado por el homicidio, la eugenesia, el encierro, la mendicidad y la marginación (3) hasta el modelo médico rehabilitador, en el cual la discapacidad es considerada una enfermedad (4), ha prevalecido una mirada de segregación sobre el colectivo de PcD. 
En la perspectiva del modelo médico rehabilitador, la PcD encarna la enfermedad, las anomalías o las deficiencias y en consecuencia debe ser atendida en instituciones especializadas que suplan sus necesidades básicas. Esta perspectiva produce unas relaciones de dominación, en las que el personal sanitario es el que decide, en gran medida, la vida que debe llevar la PcD: qué y dónde estudiar, dónde vivir, si puede o no trabajar, qué tipo de trabajo puede realizar y si puede o no procrear (5) con un claro énfasis en la dependencia, la compasión, el miedo y las actitudes paternalistas (6). Durante la primera mitad del siglo $\mathrm{xx}$, este modelo se fortaleció debido a que era promovido por organismos internacionales.

En consonancia con las políticas mundiales, Colombia también asumió y estructuró la atención a la población general con discapacidad a la luz del modelo médico rehabilitador: a finales de la década del sesenta creó el Consejo Nacional de Rehabilitación (7).

En lo que tiene que ver con la fuerza pública, para nadie es desconocido que los conflictos internos de Colombia en las últimas décadas dejan, entre muchas de sus secuelas, un número importante de sus miembros con discapacidad, como ya se señaló. El proceso de rehabilitación de esta población se realizaba a través del sistema sanitario propio de la fuerza pública sin desarrollos particulares dentro de ella. A partir de la década del setenta, toma cada vez mayor fuerza la reivindicación de los derechos de las PcD mediante movimientos sociales y llamados de la comunidad internacional. Ante esta realidad, surgió la necesidad y la urgencia de reivindicar los derechos de la PcD de la fuerza pública en particular, así que el tema se incluyó en la agenda nacional y se materializó en 1981 con la creación del Sistema Nacional de Rehabilitación (8). A pesar de que para esta época la comunidad internacional reclamaba cada vez con más fuerza la reivindicación de los derechos de las PcD y el modelo médico rehabilitador había dado un giro hacia el modelo social (9), el Sistema Nacional de Rehabilitación se creó y fundamentó en los lineamientos del modelo médico rehabilitador debido a los desarrollos propios del contexto colombiano, lo cual implicó que las personas que adquirían una discapacidad durante el cumplimiento de sus funciones recibían la atención médica necesaria, ingresaban a un programa de rehabilitación física y posteriormente eran pensionados por invalidez (10). Lamentablemente, la visión de discapacidad de este modelo lleva implícita la vulneración de los derechos de las $\mathrm{PcD}$, ya que solo se enfoca en el tratamiento clínico de la enfermedad o lesión, sin avanzar hacia un proceso de rehabilitación integral que favorezca su inclusión social.

En el siglo xxi la construcción de la política de discapacidad del sector defensa hizo la transición del modelo médico rehabilitador al modelo social, por lo que su esencia se dirigió a construir y desarrollar estrategias que permitan la integración y la inclusión social de las PcD.

Este giro implica un cambio en la comprensión de la discapacidad como un producto de los factores del medio ambiente y la organización de la sociedad mas no de la persona; así, esta resulta de las barreras sociales, la actitud y el entorno que impiden a las personas participar plenamente en la sociedad. Esta nueva perspectiva exige un cambio en las condiciones sociales para dar cabida a las necesidades de las PcD y brindarles las oportunidades que garanticen su acceso a la educación, el empleo y el ocio, es decir al goce de una vida plena (11).

Adicional al cambio de modelo, la ratificación por parte del Estado colombiano en 2009 de la Convención sobre los Derechos de las Personas con Discapacidad de Naciones Unidas 
(12) favoreció el desarrollo legislativo, político y de programas orientados al cumplimiento de los postulados de la convención, lo cual implicó avanzar en la inclusión como estrategia para el reconocimiento de sus derechos fundamentales y facilitar los medios para el desarrollo de los proyectos de vida de las PcD de las fuerzas armadas.

Existen pocas experiencias en el ámbito mundial que den cuenta de un interés particular por las PcD de la fuerza pública. En Europa, está el caso de España que en 2016 creó la Oficina de Atención a la Discapacidad en las Fuerzas Armadas para informar y asesorar al personal militar para su integración a la sociedad, con fundamento en el modelo social y en consonancia con los lineamientos de la Convención Internacional de Derechos de las Personas con Discapacidad de Naciones Unidas (13).

En Asia, se destaca la experiencia de Corea que después de la guerra creó el Centro Nacional de Rehabilitación, que desarrolla un programa de reintegración social para las PcD de sus fuerzas armadas, que incluye tratamiento médico, investigación, soporte y servicios de educación para la rehabilitación (14).

Los Estados Unidos tienen la experiencia de más larga data con el Programa de Veteranos del gobierno que atiende a las personas con discapacidad que pertenecen o pertenecieron a las fuerzas militares. Este programa se encuentra totalmente dirigido a la inclusión social sin dejar de lado la rehabilitación funcional, en este sentido, se conjugan los gustos, habilidades e intereses de las $\mathrm{PCD}$ y la destinación de recursos para su materialización. Dentro de los beneficios a los que pueden acceder los veteranos se destacan: oportunidades de educación, subsidio por compensación, rehabilitación profesional y empleo, y acondicionamiento de vivienda, entre otros. El programa es apoyado por diferentes empresas y fundaciones civiles norteamericanas (15).

En América Latina, los derechos de las PcD de las fuerzas armadas se encuentran incorporados en la política general de los países y no se conocen desarrollos específicos para esta población. Un ejemplo de ello es Ecuador, que en la Agenda Nacional para la Igualdad en Discapacidades 2013-2017, incluyó en uno de sus apartados a las fuerzas armadas y de policía para precisar que la prestación de servicios sanitarios se realiza a través de instituciones estatales como la Junta de Beneficencia, el Instituto de Seguridad Social de las Fuerzas Armadas (ISSFA) y el Instituto de Seguridad Social de la Policía Nacional (ISSPOL), pero reconoce que no cuentan con un plan de atención que integre los diferentes aspectos del aseguramiento y concesión de prestaciones dirigidos a esta población (16).

Es así que el caso de la política de discapacidad para la fuerza pública en Colombia resulta un hecho destacable en el concierto latinoamericano y mundial, a pesar de las limitaciones que pueda presentar en su formulación e implementación.

Planeación, implementación e impacto de la política en los derechos humanos de las PcD de la fuerza pública

Los cambios más importantes en materia de política para las PcD en el sector seguridad y defensa del Estado colombiano han sido coyunturales y como respuesta a las políticas nacionales para la población general. Es así que solo hasta el año 2002, el MDN comenzó a participar en el Plan Nacional de Discapacidad 2002-2006 (17), que permitió un avance significativo: primero, la reforma al régimen de pensiones de invalidez en las fuerzas militares, a través de la equiparación a la norma del Sistema General de Seguridad Social en Salud (sgsss), mediante la 
cual se pasó del $75 \%$ al $50 \%$ de la calificación de pérdida de aptitud psicofísica, lo cual permitió a un número importante de sus miembros mantener el vínculo laboral y a todos, en su conjunto, tener acceso a salud, educación y programas sociales que permiten la inclusión de las personas y sus familias (18). Segundo, se aprobó la Directiva Ministerial de Políticas en Discapacidad para el personal de la fuerza pública ${ }^{3}$, en la que se ordenó la construcción de la política pública para el sector, siguiendo el enfoque de rehabilitación integral (19). Por último, se crearon las Unidades de Rehabilitación Integral (URIS) para lograr la capacitación y ubicación laboral efectiva del personal militar con discapacidad a causa del combate en concordancia con el modelo social (17).

Las URIs fueron planteadas como grupos organizados a nivel regional encargados de evaluar, tratar y realizar el seguimiento a las PcD. Desafortunadamente, por la falta de personal y equipos para su implementación en las regiones, las decisiones de tratamiento continuaron a cargo de una persona (médico o fisioterapeuta) lo cual impidió su desarrollo. Estas fueron reemplazadas por los equipos interdisciplinarios de rehabilitación, que mantienen las fallas estructurales en la conformación de los equipos de profesionales descritos y el personal necesario para el logro de sus objetivos.

También en 2002 la Dirección General de Sanidad Militar conformó el Área de Rehabilitación y Discapacidad encargada de liderar actividades y ejecutar el programa de rehabilitación, el cual consiste en desarrollar acciones de planeación, seguimiento y control a las direcciones de sanidad de las fuerzas armadas, las cuales a su vez coordinan los servi-

3 Por ser un documento interno de trabajo del MDN, este documento no se encuentra publicado. Puede ser consultado en la oficina de la Subdirección de Salud de la Dirección General de Sanidad Militar. cios de rehabilitación en los establecimientos de sanidad militar. Es importante destacar la elaboración y puesta en marcha del modelo de rehabilitación funcional (20), que emitió lineamientos que permiten articular el sistema dentro del modelo social de la discapacidad (17).

Simultáneamente se comenzó a estructurar la política de discapacidad del sector seguridad y defensa, la cual pretende cumplir y desplegar los objetivos de una de las principales tareas que había señalado el Conpes 3591 de 2009 (1), orientado a "mitigar la deuda social con los miembros de la fuerza pública que, en cumplimiento de la función constitucional o como consecuencia de ella, se ven en riesgo de adquirir una discapacidad o efectivamente terminan adquiriéndola" y diseñar el SGRRI (21). Adicionalmente, con la Ley 1471 se definen los alcances de la rehabilitación integral para la fuerza pública, sus fases y beneficiarios, que se determinan por un informativo administrativo que no certifica la discapacidad, sino la causa de la lesión (22).

A partir de los lineamientos del Conpes 3591 de 2009 (1), el SGRRI buscó articular acciones de rehabilitación en el sector defensa pero sus acciones fueron desorganizadas, los esfuerzos de las diferentes entidades del sector fueron repetidos y los resultados pudieron ser mejores con una dirección central (21), por lo cual empezó a plantearse la necesidad de un ordenamiento central que facilitara la articulación e integración del sistema.

Un primer avance en esta dirección consistió en definir las competencias de los actores del SGRRI (19). Sin embargo, esta directiva presentó vacíos de contenido en lo relativo a los requisitos de admisión a los procesos de inclusión, lo cual limitó de entrada su implementación. También se evidenció una falta de concordancia entre algunas misiones asignadas y las funciones de los actores implicados; además, no 
incluyó indicadores de evaluación ni fijó un cronograma de aplicación. No obstante, esta directiva representa un avance en la verificación de las acciones realizadas y en la búsqueda de mecanismos que permitan la articulación central de las medidas orientadas a la inclusión social de las PcD de la fuerza pública.

En materia de derechos, esta legislación se constituye en el mayor avance y es el piso jurídico con que cuentan las $\mathrm{PcD}$ para hacerlos efectivos. Sin embargo, la Ley 1471 presenta un vacío pues al no definir quién es una persona con discapacidad, limita el acceso a la fase de rehabilitación inclusiva. Para la prestación de estos servicios, también se proyectó la construcción de un Centro de Rehabilitación Integral para lo cual se firmó, en octubre de 2010, un convenio de cofinanciación con el gobierno de Corea del Sur (23).

En cumplimiento de esta normativa, se avanzó en la construcción de la política de discapacidad para la fuerza pública mediante mesas de trabajo que funcionaron según los principios del modelo social de la discapacidad y se enmarcaron en los lineamientos del Conpes 3591 de 2009 (1).

La construcción de esta política contó con la participación de entidades del sector defensa, entidades públicas y organizaciones sociales. Los principios rectores que guían el desarrollo de la política son la dignidad humana, la corresponsabilidad, la accesibilidad, la igualdad de oportunidades, la no discriminación, la visión holística de la discapacidad, el enfoque diferencial, la interrelación sistémica, el enfoque en la prevención y previsión, la adaptabilidad, la planeación y la sostenibilidad, todos coherentes con la Convención de Personas con Discapacidad de Naciones Unidas.

Finalmente, la política de discapacidad del sector seguridad y defensa fue adoptada mediante Resolución 4584 de 2014 (24), con el fin de articular los esfuerzos de dicho sector y de otras entidades públicas y privadas en el tema de la prevención de la discapacidad y el desarrollo del SGRRI, el cual se divide en tres fases: previsión, prevención y protección; rehabilitación funcional, y rehabilitación inclusiva.

En materia de derechos humanos, es importante resaltar el gran avance del sector defensa en la visión y manejo de la discapacidad y la rehabilitación en tan corto tiempo, con una normativa robusta, orientada a la plena inclusión social de las PcD de la fuerza pública. El MDN ha sido uno de los pocos que sin tener dentro de sus funciones la rehabilitación de las PcD diseñó, implementó y lideró dicha política con la destinación de los recursos financieros y humanos necesarios.

Sin embargo, las condiciones y el contexto en el que se desarrollan estas iniciativas ameritan el análisis de los avances y las limitaciones que esta política ha tenido en materia de derechos fundamentales.

Avances en la implementación de la politica de discapacidad

La creación de la política de discapacidad es un buen punto de partida de un largo proceso para lograr la inclusión social de las PcD de la fuerza pública, que requiere de la integración y concientización de sectores privados y públicos para generar estrategias que permitan ampliar oportunidades educativas, políticas, económicas, sociales y ocupacionales para estas personas.

Los avances más importantes para la promoción, prevención y protección están en la creación de batallones de desminado para prevenir la discapacidad: en el departamento de Caldas, se han desactivado 366 minas y se han despejado 50 veredas en el municipio de Samaná (25). 
También se han desarrollado estrategias de sensibilización social como la celebración del día Remángate y la Carrera Matamoros para crear conciencia nacional sobre el efecto de las minas antipersonales. Como producto de esta sensibilización, cada vez más personas y empresas nacionales e internacionales se unen a causas para recordar y promover los derechos de las PcD víctimas de minas y aportar recursos para su rehabilitación.

En materia de salud ocupacional, se adelantan acciones orientadas a disminuir accidentes laborales que puedan llevar a una discapacidad y se han incrementado las de promoción de la salud que disminuyen la incidencia de patologías que conlleven a una discapacidad. En la misma línea de la prevención, se desarrollan campañas institucionales con la participación de altos mandos militares, que tienen como fin minimizar novedades fuera de combate. También se han iniciado acciones piloto de promoción en salud y educación en discapacidad, en la escuela de soldados profesionales que le permiten a los miembros de la fuerza pública conocer los riesgos a los que se encuentran expuestos y lo que deben hacer en caso de adquirir una discapacidad.

En relación con los procesos de rehabilitación, se evidencia mejoría en cuanto a la oportunidad, construcción y aplicación de modelos de atención, como en el caso particular de la protetización que solo se realizaba en Bogotá, que gracias a la descentralización de la atención en el Subsistema de Salud de las Fuerzas Militares y Policía Nacional (sSfmpN) ha disminuido el tiempo en el cambio de la prótesis o sus partes.

Otro de los avances de la política tiene relación con los programas de actividad física, deporte, recreación y cultura que ofrece el sector defensa que son de interés para las $\mathrm{PcD}$ y sus familias como proyecto de vida y como complemento a sus actividades.

Finalmente, la creación del CRI ha sido fundamental para avanzar en la rehabilitación inclusiva pues allí se adelantan procesos de rehabilitación integral, centrados en el proyecto de vida de la persona, con infraestructura y tecnología de punta.

En América Latina no existe una institución que pueda compararse con las dimensiones y servicios prestados en el CRI, que es el primer centro en Colombia dirigido a los miembros de la fuerza pública, en el que se pueden encontrar en un solo espacio diferentes servicios dirigidos a la inclusión. Sin embargo, es preciso señalar que la creación y puesta en marcha del CRI se produjo en medio del conflicto armado entre el Estado y las guerrillas, a causa del cual el número de $\mathrm{PcD}$ de las fuerzas crecía permanentemente. La firma del acuerdo de paz con las Fuerzas Armadas Revolucionarias de Colombia (FARC) implica una reducción sustancial de víctimas y de PcD, por lo que resulta indispensable redimensionar este centro para aprovechar al máximo su capacidad. Esto supone una revisión de la normativa que permita que personas diferentes a las de la fuerza pública se beneficien de sus servicios.

Limitaciones en la implementación de la política de discapacidad

La política de discapacidad del sector seguridad y defensa define la discapacidad de acuerdo al modelo social y a la Calificación Internacional del Funcionamiento de la Discapacidad y de la Salud - CIF- (26); sin embargo, existe la gran falencia que en ningún documento legal se especifica quién califica o determina quién presenta una discapacidad. Lo anterior ha generado grandes vacíos jurídicos para acceder a los beneficios de la política y las leyes. 
Dentro de los principios rectores para la implementación de la política (26) se encuentra la accesibilidad, la igualdad de oportunidades y la no discriminación, que busca la concordancia con la Ley 1346 de 2009 (12). En este sentido las entidades del sector deben trabajar en habilitación para adaptarse al diseño universal, para mejorar las condiciones del personal con discapacidad, esto debe incluir transporte, infraestructura de tecnologías de la información y la comunicación (braille, lenguaje de señas, etc.).

Teniendo en cuenta lo anterior, y para la exitosa implementación de la política, es necesaria la participación de los beneficiarios en la construcción de la misma. Al respecto Vásquez sostiene que la información debe ser accequible a todos, especialmente a las $\mathrm{PcD}$, de acuerdo al principio de igualdad de derechos en el que las necesidades de cada uno tienen la misma importancia y sobre el cual deben planificarse las sociedades y los recursos, de forma que se asegure que todas las personas tengan igual oportunidad de participación (27).

Es claro que la primera limitación en el diseño de la política fue la exclusión de las PcD de la fuerza pública como actores clave del proceso; lo anterior se evidenció en que no se realizó una convocatoria de participación ni para ellos ni para sus familias en las mesas de trabajo donde se elaboró y discutió dicha política.

Por otro lado, si se acepta que los contenidos de una política deben ser totalmente accesibles a la población - en especial a las $\mathrm{PcD}$, que en este caso serían las beneficiadas de la misma- se evidencia una limitación de su alcance. En este sentido, la política de discapacidad del sector seguridad y defensa no resulta accesible dado que la mayoría de los involucrados en el SGRRI desconocen los contenidos de la política por falta de difusión mediante mecanismos dirigidos a las personas con diferentes tipos de discapacidad (las principales acciones van dirigidas a la población de las fuerzas armadas con discapacidad física, la política no está disponible en audio, lenguaje braille u otros medios para que todas las personas tengan acceso a esta y les sea de fácil entendimiento).

$\mathrm{Al}$ respecto, la Organización de Naciones Unidas reconoce que la accesibilidad es un tema que afecta a todos y es el punto más álgido para lograr la transición del modelo médico al social para promover la igualdad de oportunidades para todos, por lo que las publicaciones y los entornos deben ser de fácil acceso (28).

En este mismo sentido, Diniz sostiene que una PcD afronta discriminación y desigualdad en función del grado de accesibilidad de una sociedad. Cuanto mayor sean las barreras sociales, mayores serán las restricciones de participación impuestas a los individuos con deficiencias (29).

Otro problema referido a la accesibilidad de la política, contemplado en el literal 5.2.2 de la Política de Discapacidad del Sector Seguridad y Defensa, se relaciona con la falta de programas y equipos interdisciplinarios, y la persistencia de la habitual atención individual, sin el apoyo de otras especialidades. Es necesario reconocer que con el trabajo interdisciplinario se obtienen mejores resultados y se logra un éxito rotundo (27). Una de las principales fallas de no contar con estos equipos interdisciplinarios es el acompañamiento emocional y psicológico, pues la rehabilitación se centra únicamente en la dimensión física y no hay seguimiento ni apoyo para conocer su impacto después de culminada.

Así mismo, a pesar de estar contemplado en la política en el numeral 5.1.1, una de las problemáticas más sensibles que enfrentan las $\mathrm{PcD}$ de la fuerza pública radica en la falta de orientación al momento de la lesión que permita conocer cuál va a ser la ruta y el proceso de rehabilitación $\mathrm{y}$ los derechos que adquieren por presentar una discapacidad (pensión, educación y trabajo, entre otros), pues no existe un programa centralizado que ofrezca toda la información pertinente. 
Nuevamente, se observa cómo la accesibilidad, en este caso a la información, continúa vulnerando los derechos.

En relación con la movilidad, en concordancia con el numeral 4.6 de la política en el que se busca garantizar una infraestructura adecuada con diseño universal, vale la pena mencionar que la mayoría de las instalaciones dispuestas para la atención de las PcD de la fuerza pública son antiguas y presentan numerosas barreras de acceso, en especial en áreas comunes, como escaleras, ascensores, baños y parqueaderos. Subsanar este problema de accesibilidad supone una inversión importante. Para superar este impasse es necesario tener en cuenta las normas universales desde las etapas iniciales de los proyectos (27).

Otro aspecto débil se relaciona con el grado de incorporación de la familia al proceso de rehabilitación. Así como la Convención sobre los Derechos de las Personas con Discapacidad de Naciones Unidas puntualiza el compromiso de asistencia y apoyo para las personas con discapacidad, sus familiares y cuidadores, por lo cual se les debe brindar información y educación (30), la política en su numeral 5.2.2.iii menciona el apoyo de la familia como eje principal en la rehabilitación. En la aplicación de la política para la fuerza pública, la familia no es tenida en cuenta en el proceso de rehabilitación y los cuidadores no reciben ningún tipo de capacitación para el acompañamiento de la PcD.

Si bien resulta evidente la limitación en los programas de capacitación para mejorar las relaciones personales, familiares, laborales y de pareja, se hace necesario resaltar el trabajo de los centros de familia ${ }^{4}$ que ya trabajan para estructurar este apoyo integral.

4 Los centros de familia del ejército nacional son los encargados de generar estrategias de promoción, prevención, activación de redes de apoyo y orientación dirigidas a las familias y personal sensible (heridos en combate, secuestrados, desaparecidos,
En el tema laboral, a pesar de que la Ley 1618 de 2013 promueve la empleabilidad de las PcD (31) ni la empresa privada ni el sector defensa cumplen con un porcentaje significativo de inclusión laboral. En Colombia, el 90\% de las compañías no cuentan con empleados que tengan alguna discapacidad, lo que lo hace uno de los países con menor número de $\mathrm{PcD}$ trabajando en comparación con Chile (78\%), México (65\%), Argentina (63\%) y España (56\%) (32). Por su parte, en el MDN prevalece el criterio de pensionar a la persona que en cumplimento de su deber adquiere la discapacidad, frente a la posibilidad de adecuar los ambientes de trabajo o reubicarlos laboralmente (actividades administrativas) para que continúen vinculados a la institución; esto se traduce en la ausencia de programas para que las $\mathrm{PcD}$ puedan acceder al mercado laboral, ya sea a través de ofertas de empleo formal o informal. En términos generales, al personal de soldados se le da la baja ${ }^{5}$, mientras que para oficiales y suboficiales, la discapacidad se convierte en un obstáculo para ascender, ya que no se tienen en cuenta los parámetros habituales de evaluación, sino que el análisis se centra en la deficiencia o limitación funcional. En los dos casos resulta explícita la vulneración de derechos, con mayor discriminación por rango.

Para la población pensionada de la fuerza pública, la Ley 973 de 2005 contempla el modelo de héroes, que ofrece un subsidio para solucionar vivienda de interés social, con mejoras; desafortunadamente las viviendas entregadas no cumplen los estándares de accesibilidad para las $\mathrm{PcD}(33)$.

detenidos, viudas y huérfanos) de la unidad operativa menor con el fin de mejorar las condiciones de vida a este personal.

5 Término utilizado para referirse al proceso de desvinculación laboral en las fuerzas militares de Colombia y la policía nacional. 


\section{Conclusiones y recomendaciones}

Se puede concluir que el Estado colombiano es el único país de América Latina que cuenta con una política estructurada de discapacidad para los miembros de la fuerza pública, como consecuencia del conflicto armado interno acaecido en los últimos 60 años, que lo obligó a desarrollar programas para la atención de las PcD.

Esta política fue diseñada con base en el modelo social, el cual busca que sea la sociedad la responsable de la inclusión, por lo cual se debe eliminar cualquier barrera de accesibilidad que no permita este objetivo. En este sentido, vale la pena precisar que la accesibilidad es el derecho que en la actualidad se encuentra más vulnerado y no permite la inclusión social, que se evidencia en la falta de accesibilidad a la información, educación, salud y libre movilidad por las barreras arquitectónicas; al ser este el punto más álgido es el que requiere una intervención profunda e inmediata por parte del Estado colombiano y las PcD.

Para que una política tenga un impacto positivo y cumpla el objetivo para la cual fue construida debe incluirse desde las primeras fases de su construcción a todos los actores involucrados en la misma, en este caso, las personas con discapacidad deben aportar desde sus experiencias y necesidades.

Al comenzarse a consolidar el proceso de paz se ha observado una significativa disminución de victimas militares por causa del conflicto, pero no se puede olvidar que la discapacidad también se genera por factores prevenibles en los que se debe continuar trabajando para minimizar los riesgos en las primeras fases del SGRRI.

A pesar de que aún se presentan limitaciones en cuanto al goce de derechos fundamentales como la salud, la educación y el empleo, entre otros, es claro que el esfuerzo del MDN ha comenzado a dar sus primeros frutos en cuanto a la implementación de la política, plasmada en la articulación del SGRRI. Se valora positivamente que la comprensión de la discapacidad y la rehabilitación han evolucionado a un enfoque de derechos.

\section{Agradecimientos}

Agradecimientos especiales por su aporte incondicional y su asesoría permanente al Dr. Israel Cruz Velandia, Fisioterapeuta y doctor en Salud Colectiva; a la Dra. Lilia Virginia García, Fisioterapeuta, magíster en Antropología Social; a la Dra. Paula Comellas Angulo, Licenciada en Derecho y doctora en Derecho Internacional. A todos y cada uno de los héroes de la fuerza pública de Colombia que han perdido su vida o han adquirido una discapacidad en cumplimiento del deber por sus aportes invaluables a esta reflexión.

\section{Descargos de responsabilidad}

Las opiniones expresadas en este artículo son propias y no posición oficial de la institución.

\section{Conflicto de intereses}

Ninguno declarado por la autora. El diseño, análisis y revisión del presente manuscrito fue responsabilidad de la autora. 


\section{Tabla 1. Normatividad sobre discapacidad en Colombia}

\begin{tabular}{|c|c|c|}
\hline Norma & Año & Concepto \\
\hline Decreto 3132 & 26/12/1968 & $\begin{array}{l}\text { Por el cual se crea el Consejo Nacional de Rehabilitación como entidad asesora del } \\
\text { Gobierno adscrito al Instituto Colombiano de Seguros Sociales y se dictan otras } \\
\text { disposiciones sobre rehabilitación y empleo de inválidos. }\end{array}$ \\
\hline Decreto 2358 & 28/08/1981 & Por el cual se coordina el Sistema Nacional de Rehabilitación. \\
\hline Ley 10 & $10 / 01 / 1990$ & $\begin{array}{l}\text { Por la cual se reorganiza el Sistema Nacional de Salud y se dictan otras disposicio- } \\
\text { nes; inclusión proceso rehabilitación en sistema de salud }\end{array}$ \\
\hline \multirow{5}{*}{$\begin{array}{l}\text { Constitución } \\
\text { Política }\end{array}$} & \multirow{5}{*}{ 04/07/1991 } & Artículos 13, 47, 54 y 68 \\
\hline & & $\begin{array}{l}\text { Artículo 13. Todas las personas nacen libres e iguales ante la ley, recibirán la mis- } \\
\text { ma protección y trato de las autoridades y gozarán de los mismos derechos, liberta- } \\
\text { des y oportunidades sin ninguna discriminación por razones de sexo, raza, origen } \\
\text { nacional o familiar, lengua, religión, opinión política o filosófica. }\end{array}$ \\
\hline & & $\begin{array}{l}\text { Artículo } 47 \text {. El Estado adelantará una política de previsión, rehabilitación e } \\
\text { integración social para los disminuidos físicos, sensoriales y psíquicos, a quienes se } \\
\text { prestará la atención especializada que requieran. }\end{array}$ \\
\hline & & $\begin{array}{l}\text { Artículo 54. Es obligación del Estado y de los empleadores ofrecer formación } \\
\text { y habilitación profesional y técnica a quienes lo requieran. El Estado debe } \\
\text { propiciar la ubicación laboral de las personas en edad de trabajar y garantizar a los } \\
\text { minusválidos el derecho a un trabajo acorde con sus condiciones de salud. }\end{array}$ \\
\hline & & $\begin{array}{l}\text { Artículo 68. Los particulares podrán fundar establecimientos educativos. La ley } \\
\text { establecerá las condiciones para su creación y gestión. }\end{array}$ \\
\hline Ley 188 & 1995 & $\begin{array}{l}\text { Plan Nacional de Desarrollo e Inversiones: inclusión discapacidad plan nacional de } \\
\text { desarrollo }\end{array}$ \\
\hline Ley 1346 & $31 / 07 / 2009$ & $\begin{array}{l}\text { Por medio de la cual se aprueba la "Convención sobre los Derechos de las personas } \\
\text { con Discapacidad", adoptada por la Asamblea General de la Naciones Unidas el } 13 \\
\text { de diciembre de } 2006 .\end{array}$ \\
\hline Ley 1618 & $27 / 02 / 2013$ & $\begin{array}{l}\text { Por medio de la cual se establecen las disposiciones para garantizar el pleno ejerci- } \\
\text { cio de los derechos de las personas con discapacidad }\end{array}$ \\
\hline Ley 923 & $30 / 12 / 2004$ & $\begin{array}{l}\text { Mediante la cual se señalan las normas, objetivos y criterios que deberá observar el } \\
\text { Gobierno Nacional para la fijación del régimen pensional y de asignación de retiro } \\
\text { de los miembros de la Fuerza Pública de conformidad con lo establecido en el artí- } \\
\text { culo 150, numeral 19, literal e) de la Constitución Política. }\end{array}$ \\
\hline $\begin{array}{c}\text { Directiva } \\
\text { Ministerial } 23\end{array}$ & 2005 & $\begin{array}{l}\text { 1. Directiva Ministerial de Políticas en Discapacidad para el personal de la Fuerza } \\
\text { Pública, en la que se ordenó la construcción de la política pública para el sector, bajo } \\
\text { el enfoque de rehabilitación integral. }\end{array}$ \\
\hline $\begin{array}{c}\text { Directiva } \\
110101\end{array}$ & 2006 & $\begin{array}{l}\text { Crearon las Unidades de Rehabilitación Integral (URIS), para lograr la capacitación } \\
\text { y ubicación laboral efectiva del personal militar con discapacidad a consecuencia } \\
\text { del combate, concordante con el modelo social }\end{array}$ \\
\hline $\begin{array}{l}\text { Resolución } \\
1434\end{array}$ & 2006 & $\begin{array}{l}\text { Por la cual se conforma el Área de Rehabilitación y Discapacidad, encargada de li- } \\
\text { derar estas actividades, y propuso el programa de rehabilitación militar }\end{array}$ \\
\hline Ley 1471 & $30 / 06 / 2011$ & $\begin{array}{l}\text { Por medio de la cual se dictan normas relacionadas con la rehabilitación integral de los } \\
\text { miembros de la Fuerza Pública, alumnos de las escuelas de formación de las Fuerzas Mi- } \\
\text { litares y sus equivalentes en la Policía Nacional, personal civil del Ministerio de Defensa } \\
\text { Nacional y de las Fuerzas Militares y personal no uniformados de la Policía Nacional. }\end{array}$ \\
\hline $\begin{array}{l}\text { Conpes Social } \\
3591 \\
\end{array}$ & 2009 & Estructuración de la política de discapacidad \\
\hline $\begin{array}{c}\text { Resolución } \\
4584\end{array}$ & 2014 & Adopción de la Política de Discapacidad Sector Seguridad y Defensa \\
\hline
\end{tabular}




\section{Referencias}

1. Colombia, Vicepresidencia de la República, Ministerio de Defensa Nacional, Ministerio de la Protección Social, Departamento Nacional de Planeación, sena. Conpes 3591, Sistema de rehabilitación integral para la Fuerza Pública [internet]. 2009 [citado 2016 oct 20]. Disponible en: https://colaboracion.dnp. gov.co/CDT/CONPES/Econ \%C3\%B3micos/3591.pdf

2. Colombia, Ministerio de Defensa Nacional. Reporte de la oficina de Bienestar Sectorial y Salud. Documento interno de trabajo. 2015.

3. Palacios A. El modelo social de discapacidad: orígenes, caracterización y plasmación en la Convención Internacional sobre los Derechos de las Personas con Discapacidad. Madrid: Grupo Editorial Cinca; 2008.

4. Velarde-Lizama V. los modelos de la discapacidad: un recorrido histórico. Empresa y Humanismo. 2012;15(1),115-36.

5. Rieser RI. Inclusive Education or special educational needs: Meeting the challenge of disability discrimination in schools. Londres: Routledge; 2006.

6. Céspedes G. La nueva cultura de la discapacidad y los modelos de rehabilitación. Aquichán, 2005;5(1):108-13.

7. Colombia, Presidencia de la República, Decreto 3132, Consejo Nacional de Rehabilitación como entidad asesora del Gobierno adscrito al Instituto Colombiano de Seguros Sociales y se dictan otras disposiciones sobre rehabilitación y empleo de inválidos (1968 dic 26).

8. Colombia, Presidencia de la República, Decreto 2358, Sistema Nacional de Rehabilitación (1981).

9. Campoy I. (Ed). Los derechos de las personas con discapacidad. Perspectivas sociales, políticas, jurídicas y filosóficas, Madrid: Carlos III-Dykinson; 2004.

10. Colombia, Ministerio de Defensa Nacional. Memorias al congreso 1947.

11. Gannon B \& Nolan B. Disability and social inclusion in Ireland. [internet]. 2007 [citado 2016 abr 04]. Disponible en: http://www.ihrec.ie/download/pdf/disability_and_social_inclusion_in_ireland.pdf

12. Colombia, Congreso de la Republica. Ley 1346, Por medio de la cual se aprueba la convención sobre los derechos de las personas con discapacidad, adoptada por la Asamblea General de las Naciones Unidas el 13 de diciembre de 2006 (2009 jul 31).

13. España, Ministerio de Defensa, Oficina de Atención a la Discapacidad en las Fuerzas Armadas. Orden DEF/83/2016. Boletín Oficial de Estado 2016;(28).

14. The Nacional Rehabilitation Center, Departament of Public Medical Rehabilitation. Public Medica Rehabilitation [internet]. 2016 [citado 2017 jun 06] disponible en: http://www.ncs.go.kr

15. U.S Department of veterans affairs. Veterans Choice Program Interactive Timeline [internet]. 2014 [citado 2017 jun 06] disponible: www.va.gov

16. Consejo Nacional de la Igualdad de Discapacidades Conadis. Agenda Nacional para la Igualdad en Discapacidades 2013-2017. Cambiando vidas. Quito: Consejo Nacional de la Igualdad de Discapacidades. [internet]. 2013 [citado 2017 jun 06] Disponible en: http://www.planificacion.gob.ec/wp-content/ uploads/downloads/2014/09/Agenda-Nacional-para-Discapacidades.pdf

17. Colombia, Gobierno Nacional de la Republica. Plan Nacional de Discapacidad 2002-2006 [internet]. 2002 [citado 2016 oct 18]. Disponible en: http://www.urosario.edu.co/urosario_files/12/12e61953332f-4fc3-97d7-9df286c7c22e.pdf

18. Colombia, Congreso de la República. Ley 923, Mediante la cual se señalan las normas, objetivos y criterios que deberá observar el Gobierno Nacional para la fijación del régimen pensional y de asignación 
de retiro de los miembros de la Fuerza Pública de conformidad con lo establecido en el artículo 150, numeral 19, literal e) de la Constitución Política. (2004 dic 30).

19. Colombia, Ministerio de Defensa Nacional. Directiva Ministerial de Políticas en discapacidad para el personal de la Fuerza Pública. 2005(23). Documento interno de trabajo.

20. Colombia, Dirección General de Sanidad Militar. Modelo de rehabilitación funcional para el subsistema de salud de las Fuerzas Militares [internet]. 2012 [citado 2017 may 15] Disponible en: http://www. disanejercito.mil.co/index.php?idcategoria=166309

21. Colombia, Ministerio de Defensa Nacional. Memorias al Congreso 2012-2013 [internet]. [Citado 2016 oct 20]. Disponible en: https://www.mindefensa.gov.co/irj/go/km/docs/Mindefensa/Documentos/ descargas/Prensa/Documentos/memorias2012-2013.pdf

22. Colombia, Congreso Nacional de la República. Ley 1471, Por medio de la cual se dictan normas relacionadas con la rehabilitación integral de los miembros de la Fuerza Pública, alumnos de las escuelas de formación de las Fuerzas Militares y sus equivalentes en la Policía Nacional, personal civil del Ministerio de Defensa Nacional y de las Fuerzas Militares y personal no uniformado de la Policía Nacional (2011).

23. Colombia, Ministerio de Defensa Nacional. Informe del Ministro al Congreso Nacional de la República [internet]. 2010-2011 [citado: 2016 oct 20]. Disponible en: https://www.mindefensa.gov.co/irj/go/km/ docs/Mindefensa/Documentos/descargas/Prensa/Documentos/memorias2010-2011.pdf

24. Colombia, Ministerio de Defensa Nacional. Resolución 4584, Por la cual se adopta la política de discapacidad del sector seguridad y defensa y se dictan otras disposiciones (2014 jun 6).

25. Colombia, Oficina de Prensa Octava Brigada. Con éxito avanza labor de desminado humanitario gracias al Ejército en Caldas [internet]. 2017 [citado 2017 may 5]. Disponible en: https://ejercito.mil. $\mathrm{co} /$ ?idcategoria $=410392$

26. Colombia, Ministerio de Defensa Nacional. Política de Discapacidad del Sector Seguridad y Defensa [internet]. 2014 [citado 2016 may 12]. Disponible en: http://www.disanejercito.mil.co/index.php?idcategoria $=29653$

27. Vázquez Barrios A, Cáceres N. El abordaje de la discapacidad desde la atención primaria de la salud. Buenos Aires: Organización Panamericana de la Salud: 2008.

28. Astorga Gatjens LF. ¡Por un mundo accesible e inclusivo! Guía Básica para comprender y utilizar la Convención sobre los derechos de las personas con discapacidad [internet]. 2007 [citado 2017 jun 06]. Disponible en: www.un.org/esa/socdev/enable/documents/conventionguideesp.doc

29. Diniz D, Barbosa L, Rufino dos Santos W. Discapacidad, derechos humanos y justicia, Revista Internacional de Direitos Humanos. 2009 dic;6(11):65-77.

30. Eroles C, Fiamberti H. Los derechos de las personas con discapacidad. Buenos Aires: Universidad de Buenos aires: 2002.

31. Colombia, Congreso Nacional de la Republica. Ley estatutaria 1618. (2013).

32. Fundación Arcángeles. Estadística Inclusión Laboral Personas con discapacidad muestra la realidad en Colombia [internet]. 2016 [citado 03 jun 2017]. Disponible en: http://arcangeles.org/estadistica-empleabilidad-personas-discapacidad-muestra-la-realidad-colombia/

33. Colombia, Congreso Nacional de la Republica. Ley 973 (2005). 